\title{
Design of Adaptive Sliding Mode Controllers for Perturbed Nonlinear Systems with Partial Unmeasurable States and State Constraints
}

Chih-Chiang Cheng ( $\nabla$ chengcc@mail.ee.nsysu.edu.tw)

National Sun Yat-sen University College of Engineering https://orcid.org/0000-0002-0090-3037

Ting-Yu Lin

National Sun Yat-sen University College of Engineering

Yu-Kuo Li

National Sun Yat-sen University College of Engineering

\section{Research Article}

Keywords: barrier Lyapunov function, state observer, sliding mode control, adaptive control, perturbation estimator

Posted Date: November 19th, 2021

DOI: https://doi.org/10.21203/rs.3.rs-1006478/v1

License: (c) (i) This work is licensed under a Creative Commons Attribution 4.0 International License. Read Full License 


\title{
Design of Adaptive Sliding Mode Controllers for Perturbed Nonlinear Systems with Partial Unmeasurable States and State Constraints
}

\author{
Chih-Chiang Cheng · Ting-Yu Lin · Yu-Kuo Li
}

Received: date / Accepted: date

\begin{abstract}
A sliding mode control (SMC) strategy is proposed in this paper for a class of perturbed nonlinear systems with unmeasurable states and state constraints to deal with the state tracking problems. First of all, a partial states observer is designed for solving the problems due to unmeasurable states. The estimation errors will approach zero in a finite time. Secondly, based on a designed barrier Lyapunov function, one designs the sliding surface function and an adaptive sliding mode tracking controller to ensure that the states have the ability to track the desired signals. Moreover, the tracking error is capable of converging to zero in a finite time without violating the given state's constraints. Perturbation estimator and adaptive mechanisms are also utilized so that there is no need to know the upper bounds of perturbations and perturbation estimation errors. Finally, a numerical example is provided to demonstrate the feasibility of the proposed control strategy.
\end{abstract}

Keywords barrier Lyapunov function, state observer, sliding mode control, adaptive control, perturbation estimator.

\section{Introduction}

In order to increase the applicability of the designed control systems, there are two important factors one has to consider besides the stability issue. The first one is the availability of

Chih-Chiang Cheng

Dept. of Electrical Eng., National Sun Yat-Sen U., No. 70, Lien Hai

Rd., Kaohsiung, Taiwan, R.O.C.

Tel.: +886-7-5252000 ext 4133

Fax: +886-7-5254199

E-mail: chengcc@mail.ee.nsysu.edu.tw

Tin-Yu Lin

Dept. of Electrical Eng., National Sun Yat-Sen U., No. 70, Lien Hai Rd., Kaohsiung, Taiwan, R.O.C. the state variables, the second is the magnitudes of states that the controlled system can sustain. These two factors generally increase the difficulty of designing the controllers. Therefore, studying systems that have unmeasurable states and also have state constraints has become a very important issue.

In general, there are two ways to deal with the problems caused by unmeasurable states. One of which is to design the output feedback controller. This include [1-5]. The other way is to employ an observer. For controlling nonlinear systems with utilization of observer, Li et al. [6,7], Park et al. [8], Wang et al. [9], Choi and Yoo [10] and Huang [11] all made the estimation errors uniformly ultimate bounded (UUB). For solving the problems of regulation, Lei [12] utilized a high-gain predictor for a single-input singe-output (SISO) nonlinear system with state delay and finally made the estimation error asymptotically stable (A.S.). Laila [13] proposed an observer for a combustion engine test bench, and also made the estimation error A.S. Although the control methods mentioned above can handle the systems with unmeasurable states, several disadvantages are found in these systems: I. The dynamic equations of plants in [4-10, 12] have to be in strict feedback form. II. The methods proposed in [6-11] can only achieve the property of UUB. III. They cannot solve the problems of state constraints. IV. The methods proposed in $[3,4,7-9,12,13]$ need to meet Lipschitz condition.

As for solving the state constraint problems, many methods mainly relied on numerical methods or used complex algorithms. For example, invariant set method [14, 15], model predictive control [16-18], reference governor [19]. The disadvantage of these methods is their huge amount of computation, which leads to longer control time and may cause instability in the system. Therefore, lots of researches on state constraints have been developed based on establishing barrier Lyapunov functions due to its simplicity. When utiliz- 
ing a barrier Lyapunov function methodology [20-34], one needs to create a barrier Lyapunov function first, and then design the controller through the function such that not only the barrier Lyapunov function is bounded, but also the controlled system is stable.

In order to deal with unmeasurable states and state constraint problems, backstepping control which utilizing barrier Lyapunov function were proposed in [20-26] for perturbed nonlinear systems, these methods all achieved the property of UUB. Intelligent control schemes were also developed for solving unmeasurable states and state constraint problems, and they also achieved the property of UUB [2734]. Although the aforementioned works [20-34] can handle the problems of unmeasurable states and state constraints at the same time, several disadvantages are found in these systems: I. The dynamic equations with match or unmatched perturbations considered in [20-27, 29-34] have to be in strict feedback form. II. The methods proposed in [20-34] can only achieve the property of UUB. III. The methods proposed in $[20,23,25,27-31]$ may be only applied to SISO systems.

Recently Chiang and Cheng [35] proposed an adaptive output feedback sliding mode control scheme for a class of nonlinear systems with matched and unmatched perturbations to solve regulation problems. The advantages of this control scheme include (a) the dynamic equations of the system do not need to be in strict feedback form; (b) there is no need to know the upper bounds of both match and unmatched perturbations in advance; (c) the state variables can converge to zero within a finite time; (d) it can be applied to multi-input multi-output (MIMO) systems.

In this paper we extend the research of [35] and propose an adaptive SMC scheme as well as a partial state observer for a class of perturbed systems with state constraints, so that both the estimation errors of states and tracking errors can converge to zero in a finite time. This proposed control scheme not only eliminates the disadvantages I to IV mentioned above, but also has the advantages (a) to (d) addressed previously. Moreover, it also has the following advantages: (1) it has the ability to constrain the magnitudes of full states; (2) it can solve tracking problems, and all the state-tracking errors can converge to zero within a finite time and stay thereafter. A numerical example is demonstrated for showing the effectiveness of the proposed control scheme.

\section{System Descriptions and Problem Formulations}

Consider a class of MIMO nonlinear systems with matched and unmatched perturbations, whose dynamic equations are governed by

$\dot{\mathbf{x}}(t)=\mathbf{f}(\mathbf{x})+\mathbf{B u}(t)+\Delta \mathbf{p}(t, \mathbf{x}, \mathbf{u})$
$\mathbf{y}(t)=\mathbf{C x}(t)$

where $\mathbf{x}(t) \in R^{n}$ represents unmeasurable state of the system, $\mathbf{u}(t) \in R^{m}$ is the control input, $\mathbf{y}(t) \in R^{p}$ is the measurable output. The nonlinear vector $\mathbf{f}(\mathbf{x})$ is known and $\mathbf{f}(\mathbf{x})=\mathbf{0}$ if $\mathbf{x}=\mathbf{0}$. The constant matrices $\mathbf{B}$ and $\mathbf{C}$ with approximate dimensions are also known, and $\operatorname{rank}(\mathbf{B})=m, \operatorname{rank}(\mathbf{C})=p$ The unknown vector $\Delta \mathbf{p}(t, \mathbf{x}, \mathbf{u})$ can be viewed as model uncertainty, nonlinearity or external disturbance. In addition, the number of outputs is greater than or equal to the number of inputs, i.e., $p \geq m$.

The aim of this paper is to design an adaptive sliding mode tracking controller and a partial state's observer such that the following objectives are achieved:

(a) The estimation error $\tilde{\mathbf{z}} \triangleq \mathbf{z}-\hat{\mathbf{z}}$ will converge to zero in a finite time, where the states $\mathbf{z}$ and $\hat{\mathbf{z}}$ will be introduced in section 3 .

(b) The state tracking error $\mathbf{e}(t) \triangleq \mathbf{x}-\mathbf{x}_{d}(t)$ will also approach zero in a finite time, and satisfies the requirement of $\|\mathbf{e}(t)\|<2 k_{b}+\|\mathbf{W} \tilde{\mathbf{z}}\|, \forall t$; where $\mathbf{x}_{d}(t)$ is the desired tracking signal, $k_{b}>0$ is a prespecified real constant, and $\mathbf{W}$ is a matrix which will be introduced in section 3 . The notation $\|\cdot\|$ stands for the Euclidean norm of a vector or the induced two-norm of a matrix.

\section{Design of Partial States Observer}

For achieving the control objectives, we first design a new state variable as

$\mathbf{s}(t)=\mathbf{F y}(t) \in R^{m}$,

where $\mathbf{F}$ is a designed constant matrix which fulfills the matrix equation $\mathbf{B}^{g}=\mathbf{F C}$, and $\mathbf{B}^{g}=\left(\mathbf{B}^{T} \mathbf{B}\right)^{-1} \mathbf{B}^{T}$ denotes the Moore-Penrose pseudoinverse of $\mathbf{B}$. Note that if the columns of $\left(\mathbf{B}^{g}\right)^{T}$ are in the $R\left(\mathbf{C}^{T}\right)$, then matrix $\mathbf{F}$ exists, where $R(\cdot)$ symbolizes the range space of $(\cdot)$.

According to the method addressed in [35], one is able to transform (1) as

$\begin{aligned} \dot{\mathbf{z}}(t) & =\mathbf{W}^{g} \Phi_{1}(\mathbf{s}) \mathbf{s}(t)+\Delta \mathbf{p}_{1}(t, \mathbf{x}, \mathbf{u}), \\ \dot{\mathbf{s}}(t) & =\mathbf{B}^{g} \Phi_{1}(\mathbf{s}) \mathbf{s}(t)+\mathbf{B}^{g} \Phi_{2}(\mathbf{z}, \mathbf{s}) \mathbf{z}+\mathbf{u}(t)+\mathbf{B}^{g} \Delta \mathbf{p},\end{aligned}$

where $\mathbf{M}\left[\mathbf{z}^{T}(t), \mathbf{s}^{T}(t)\right]^{T}=\mathbf{x}(t), \mathbf{M} \triangleq[\mathbf{W}, \mathbf{B}], \mathbf{W} \in R^{n \times(n-m)}$ is a full rank matrix, $R(\mathbf{W}) \cap R(\mathbf{B})=\{\mathbf{0}\}, \Delta \mathbf{p}_{1} \triangleq \mathbf{W}^{g} \Phi_{2} \mathbf{z}$ $+\mathbf{W}^{g} \Delta \mathbf{p}$. The matrix $\mathbf{W}^{g}=\left(\mathbf{W}^{T} \mathbf{W}\right)^{-1} \mathbf{W}^{T}$ is the MoorePenrose pseudoinverse of $\mathbf{W}$, the two matrices $\Phi_{1}, \Phi_{2}$ are given by [35]

$$
\begin{gathered}
\Phi_{1}(\mathbf{s})=\left.\int_{0}^{1} \frac{\partial \mathbf{f}\left(\mathbf{0}, \Lambda_{1}\right)}{\partial \Lambda_{1}}\right|_{\Lambda_{1}=\mathbf{s} \rho} d \rho \in R^{n \times m}, \\
\Phi_{2}(\mathbf{z}, \mathbf{s})=\left.\int_{0}^{1} \frac{\partial \mathbf{f}\left(\Lambda_{2}, \mathbf{s}\right)}{\partial \Lambda_{2}}\right|_{\Lambda_{2}=\mathbf{z} \rho} d \rho \in R^{n \times(n-m)}
\end{gathered}
$$


Noted that the new state variable $\mathbf{z}(t) \in R^{n-m}$ is unmeasurable.

To solve the tracking problem, one firstly designs the sliding surface function $\sigma(t) \in R^{m}$ as

$\sigma(t)=\mathbf{F}\left(\mathbf{y}-\mathbf{y}_{d}\right)$

where $\mathbf{y}_{d}=\mathbf{C} \mathbf{x}_{d}$. Since the state $\mathbf{z}(t)$ is unmeasurable, one designs a partial states observer for estimating $\mathbf{z}(t)$ as

$$
\begin{aligned}
\dot{\mathbf{z}}(t)= & \mathbf{W}^{g} \Phi_{1}(\mathbf{s}) \mathbf{s}(t)-\frac{\mathbf{e}_{\hat{z}}}{\left\|\mathbf{e}_{\hat{z}}\right\|^{2}} \sin \theta_{1}\left(\mathbf{e}_{\hat{z}}\right) \cos \theta_{1}\left(\mathbf{e}_{\hat{z}}\right)+\eta_{1}(t) \\
& +\mathbf{W}^{g} \dot{\mathbf{x}}_{d},
\end{aligned}
$$

where

$$
\begin{aligned}
& \mathbf{e}_{\hat{z}}=\hat{\mathbf{z}}-\mathbf{W}^{g} \mathbf{x}_{d}, \\
& \theta_{1}=\left(\|\mathbf{W}\|^{2}\left\|\mathbf{e}_{\hat{z}}\right\|^{2} \pi\right) / 2 k_{b}^{2} .
\end{aligned}
$$

The function $\eta_{1} \in R^{n-m}$ in (5) is generated from

$\dot{\eta}_{1}(t)=\left\{\begin{array}{r}-\left[\rho_{1}+h_{1} \zeta(\mathbf{u})+\hat{c}_{0}(t)+\hat{c}_{1}(t) \beta(\hat{\mathbf{x}})\right. \\ \left.+\Psi_{1}\right] \frac{\eta_{1}(t)}{\left\|\eta_{1}(t)\right\|}, \quad \text { if } \eta_{1}(t) \neq \mathbf{0} \\ \varphi_{1} e^{-t}, \quad \text { if } \eta_{1}(t)=\mathbf{0}\end{array}\right.$

where $\rho_{1}$ is a designed positive constant, $\varphi_{1}$ is a designed vector with positive constant entries, $\Psi_{1}=\left\|\eta_{1}\right\|+\left\|\mathbf{W}^{g} \dot{\mathbf{x}}_{d}\right\|$ $+\left|\sin \theta_{1} \cos \theta_{1}\right| /\left\|\mathbf{e}_{\hat{z}}\right\|$. The adaptive gains $\hat{c}_{k}(t), k=0,1$, are computed from the following adaptive laws as

$$
\begin{aligned}
& \dot{\hat{c}}_{0}(t)= \begin{cases}0, & \text { if } \eta_{1}=\mathbf{0} \\
1, & \text { otherwise }\end{cases} \\
& \dot{\hat{c}}_{1}(t)= \begin{cases}0, & \text { if } \eta_{1}=\mathbf{0} \\
\beta(\hat{\mathbf{x}}), & \text { otherwise }\end{cases}
\end{aligned}
$$

where $\beta(\hat{\mathbf{x}})$ in $(10), h_{1}$ and the function $\zeta(\mathbf{u})$ in $(8)$ are introduced in the next section. By using the Squeeze Theorem [36] and L'Hospital's rule, one is able to verify that

$$
\lim _{\left\|\mathbf{e}_{\hat{z}}\right\| \rightarrow \mathbf{0}} \frac{\mathbf{e}_{\hat{z}}}{\left\|\mathbf{e}_{\hat{z}}\right\|} \sin \theta_{1}\left(\mathbf{e}_{\hat{z}}\right) \cos \theta_{1}\left(\mathbf{e}_{\hat{z}}\right)=0
$$

\section{Stability Analysis of Partial State Observer}

Let $\tilde{\mathbf{z}}(t)=\mathbf{z}(t)-\hat{\mathbf{z}}(t)$ be the estimation error of partial states observer. Then from (3) and (5), one can obtain

$$
\dot{\tilde{\mathbf{z}}}(t)=\Delta \mathbf{p}_{1}+\frac{\mathbf{e}_{\hat{z}}}{\left\|\mathbf{e}_{\hat{z}}\right\|^{2}} \sin \theta_{1} \cos \theta_{1}-\eta_{1}(t)-\mathbf{W}^{g} \dot{\mathbf{x}}_{d}
$$

The stability analysis of estimation error $\tilde{\mathbf{z}}(t)$ is addressed in the following theorem.
Theorem 1 Consider the dynamic equation (11). Suppose that the designed vector $\Phi_{1}(\mathbf{s}) \neq \mathbf{0}$ for some $t$, and there exist unknown positive constants $c_{0}, c_{1}$ satisfying the following inequality

$\left\|\Delta \mathbf{p}_{1}(t, \mathbf{x}, \mathbf{u})\right\| \leq c_{0}+c_{1} \beta(\hat{\mathbf{x}})+h_{1} \zeta(\mathbf{u})$

in the domain of interest, where $\beta(\hat{\mathbf{x}})$ and $\zeta(\mathbf{u})$ are known nonnegative functions, $h_{1}>0$ is also a known constant. If the partial states observer (5) with adaptive laws (8), (9) and (10) are employed, then

(a) the estimation error $\tilde{\mathbf{z}}$ will approach zero within a finite time;

(b) the state variable $\eta_{1}$ is bounded for all time;

(c) the adaptive gains $\hat{c}_{i}, i=0,1$ are all bounded, and $\hat{c}_{i}$ will converge to a finite limit respectively as $t \rightarrow \infty$.

Proof: Define a Lyapunov function candidate as

$V_{1}=\|\tilde{\mathbf{z}}(t)\|+\left\|\eta_{1}\right\|+\frac{1}{2} \sum_{k=0}^{1} \tilde{c}_{k}^{2}(t)$

where $\tilde{c}_{k}(t) \triangleq \hat{c}_{k}(t)-c_{k}$ are adaptive errors of unknown positive constants $c_{k}$ respectively, $k=0,1$. All the possible cases that may occur when computing the time derivative of $V_{1}$ are analyzed as follows.

Case 1: $\tilde{\mathbf{z}} \neq \mathbf{0}$, and $\eta_{1} \neq \mathbf{0}$

Differentiate (13) with respect to time, we obtain

$\dot{V}_{1}=\frac{\tilde{\mathbf{z}}^{T}(t)}{\|\tilde{\mathbf{z}}(t)\|} \dot{\tilde{\mathbf{z}}}(t)+\frac{\eta_{1}^{T}(t)}{\left\|\eta_{1}(t)\right\|} \dot{\eta}_{1}+\sum_{k=0}^{1} \tilde{c}_{k}(t) \dot{\tilde{c}}_{k}(t)$.

Substituting (11) into (14) and using (12) yields

$$
\begin{gathered}
\dot{V}_{1} \leq h_{1} \zeta(\mathbf{u})+c_{0}+c_{1} \beta(\hat{\mathbf{x}})+\frac{1}{\left\|\mathbf{e}_{\hat{z}}\right\|}\left|\sin \theta_{1}\left(\mathbf{e}_{\hat{z}}\right) \cos \theta_{1}\left(\mathbf{e}_{\hat{z}}\right)\right|+ \\
\left\|\eta_{1}\right\|+\left\|\mathbf{W}^{g} \dot{\mathbf{x}}_{d}\right\|+\frac{\eta_{1}^{T}(t)}{\left\|\eta_{1}(t)\right\|} \dot{\eta}_{1}+\sum_{k=0}^{1} \tilde{c}_{k}(t) \dot{\tilde{c}}_{k}(t)
\end{gathered}
$$

By using (8), (9) and (10), one is able to obtain

$\dot{V}_{1} \leq-\rho_{1}<0$.

In this case the magnitude of $V_{1}$ is bounded, which also indicates that the states $\tilde{\mathbf{z}}, \eta_{1}$, and adaptive errors $\tilde{c}_{0}, \tilde{c}_{1}$ are all bounded.

Case 2: $\tilde{\mathbf{z}} \neq \mathbf{0}$, and $\eta_{1}=\mathbf{0}$

In this case one can see that $V_{1}=\|\tilde{\mathbf{z}}\|+\frac{1}{2} \sum_{k=0}^{1} \tilde{c}_{k}^{2}$. From (9), (10) and (15), we can know that

$\dot{V}_{1} \leq h_{1} \zeta(\mathbf{u})+c_{0}+c_{1} \beta(\hat{\mathbf{x}})+\frac{1}{\left\|\mathbf{e}_{\hat{z}}\right\|}\left|\sin \theta_{1} \cos \theta_{1}\right|+\left\|\mathbf{W}^{g} \dot{\mathbf{x}}_{d}\right\|$.

In this case $\dot{V}_{1}$ may be greater or smaller than zero. According to (8), $\dot{\eta}_{1} \neq \mathbf{0}$ in this case, the trajectory of $\eta_{1}$ will cross the surface $\eta_{1}=\mathbf{0}$ immediately, and the status of the system will switch to another case where $\eta_{1} \neq \mathbf{0}$. Nevertheless, the 
states $\tilde{\mathbf{z}}, \eta_{1}$, and adaptive errors $\tilde{c}_{0}, \tilde{c}_{1}$ are all bounded due to the continuity of the controlled state variables.

Case 3: $\tilde{\mathbf{z}}=\mathbf{0}$ and $\eta_{1} \neq \mathbf{0}$

From (13), it is seen that the Lyapunov function becomes $V_{1}=\left\|\eta_{1}\right\|+\sum_{k=0}^{1} \tilde{c}_{k}^{2}(t) / 2$. Differentiate it with respect to time and using (8), (9) and (10), one obtains

$\dot{V}_{1}=-\rho_{1}-h_{1} \zeta(\mathbf{u})-c_{0}-c_{1} \beta(\hat{\mathbf{x}})-\Psi \leq-\rho_{1}<0$.

Case 4: $\tilde{\mathbf{z}}=\mathbf{0}$ and $\eta_{1}=\mathbf{0}$

In this case $V_{1}=\frac{1}{2} \sum_{k=0}^{1} \tilde{c}_{k}^{2}$. Differentiating it with respect to time and using (9), (10), one obtains

$\dot{V_{1}}=\tilde{c}_{0} \dot{\hat{c_{0}}}+\tilde{c}_{1} \dot{\hat{c_{1}}}=0$.

The previous analysis shows that $V_{1}$ is a bounded function even if the occurrence of case 2 . One is able to see that

(a) the estimation error $\tilde{\mathbf{z}}$ will approach zero in a finite time as long as $\eta_{1} \neq \mathbf{0}$

(b) the state variable $\eta_{1}$ is bounded since $\eta_{1}^{T} \dot{\eta}_{1} \leq 0$;

(c) the adaptive gains $\hat{c}_{i}, i=0,1$ are monotonically increasing and are all bounded in accordance with (9), (10) and the above analysis. Therefore, there exit finite constants $c_{i \infty}$, $i=0,1$, such that $\lim _{t \rightarrow \infty} \hat{c}_{i}=c_{i \infty}, i=0,1$ [37].

\section{Design of Adaptive Sliding Mode Tracking Controller}

By using (2), (3), (4) and (5), one can compute the time derivative of $\mathbf{e}_{\hat{z}}$ and $\sigma$ as

$\dot{\mathbf{e}}_{\hat{z}}(t)=\mathbf{W}^{g} \Phi_{1}(\mathbf{s}) \mathbf{s}(t)-\frac{\mathbf{e}_{\hat{z}}}{\left\|\mathbf{e}_{\hat{z}}\right\|^{2}} \sin \theta_{1} \cos \theta_{1}+\eta_{1}$

$\dot{\sigma}(t)=\mathbf{B}^{g} \Phi_{1} \mathbf{S}(t)+\mathbf{B}^{g} \Phi_{2} \mathbf{z}+\mathbf{u}(t)+\mathbf{B}^{g} \Delta \mathbf{p}-\mathbf{B}^{g} \dot{\mathbf{x}}_{d}$

Now we propose an adaptive sliding mode tracking controller as

$\mathbf{u}(t)=\mathbf{u}_{f}(t)+\mathbf{u}_{s}(t)$,

where

$\mathbf{u}_{f}(t)=-\varepsilon \sigma(t)-\mathbf{B}^{g} \Phi_{1}(\mathbf{s}) \mathbf{s}(t)-\eta_{2}(t)-\mathbf{p}_{e s t}+\mathbf{B}^{g} \dot{\mathbf{x}}_{d}$,

and

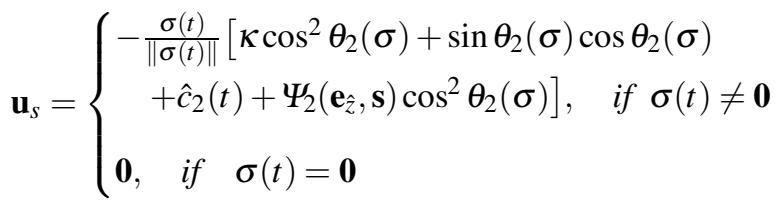

The parameters $\varepsilon$ and $\kappa$ are designed positive constants, and

$$
\begin{aligned}
\theta_{2}(\sigma) & =\frac{\|\mathbf{B}\|\|\sigma\| \pi}{2 k_{b}}, \\
\Psi_{2}\left(\mathbf{e}_{\hat{z}}, \mathbf{s}\right) & =\left(\left\|\eta_{1}\right\|+\left\|\mathbf{W}^{g} \Phi_{1}(\mathbf{s})\right\|\|\mathbf{s}(t)\|\right)\left\|\mathbf{e}_{\hat{z}}\right\| \sec ^{2} \theta_{1}\left(\mathbf{e}_{\hat{z}}\right) .
\end{aligned}
$$

The adaptive law $\hat{c}_{2}(t)$ in $\mathbf{u}_{s}$ and $\eta_{2}(t)$ in $\mathbf{u}_{f}$ are designed respectively as

$\dot{\hat{c}}_{2}(t)=\left\{\begin{array}{lll}\sec ^{2} \theta_{2}(\sigma), & \text { if } & \boldsymbol{\sigma} \neq \mathbf{0} \\ 0, & \text { if } & \boldsymbol{\sigma}=\mathbf{0}\end{array}\right.$

$\dot{\eta}_{2}(t)=\left\{\begin{array}{l}-\left[\gamma+\Psi_{2}\left(\mathbf{e}_{\hat{z}}, \mathbf{s}\right)\right] \frac{\eta_{2}(t)}{\left\|\eta_{2}(t)\right\|}, \quad \text { if } \quad \eta_{2}(t) \neq \mathbf{0} \\ \varphi_{2} e^{-t}, \quad \text { if } \quad \eta_{2}(t)=\mathbf{0}, \mathbf{e}_{\hat{z}} \neq \mathbf{0}, \sigma=\mathbf{0} \\ \mathbf{0}, \quad \text { otherwise }\end{array}\right.$

where $\gamma$ is a designed positive constant, and $\varphi_{2}$ is a designed vector with positive constant entries.

The perturbation estimation scheme proposed in [35] is also utilized in this research. Following the similar design procedure as addressed in [35], one designs a nominal signal $\sigma_{(\text {nот) }}$ and the perturbation estimation signal respectively as

$\dot{\sigma}_{(\text {nom })}=-\varepsilon \sigma_{(\text {nom })}+\mathbf{u}_{s}-\mathbf{p}_{\text {est }}$,

$\mathbf{p}_{\text {est }}(t)=\hat{\mathbf{p}}(t)+\varepsilon\left[\sigma-\sigma_{(\text {nom })}\right]$,

where $\hat{\mathbf{p}}(t)$ is the output of the derivative estimator developed in [38]. If the perturbation estimation error is defined as

$\Delta \tilde{\mathbf{p}}_{2}(t, \mathbf{x}, \mathbf{u}) \triangleq \mathbf{B}^{g} \Phi_{2}(\mathbf{z}, \mathbf{s}) \mathbf{z}(t)+\mathbf{B}^{g} \Delta \mathbf{p}-\eta_{2}-\mathbf{p}_{e s t}$,

then one can verify that

$\Delta \tilde{\mathbf{p}}_{2}(t, \mathbf{x}, \mathbf{u})=-\delta(t)$,

where $\delta(t)$ stands for the derivative estimation error. Equation (28) indicates that causing the perturbation estimation error is only due to the derivative estimation error signal $\delta(t)$. Note that if the derivative estimator is designed properly, the signal $\delta(t)$ is not only bounded, but also asymptotically stable in accordance with the analysis in [38].

\section{Stability Analysis}

The stability of the proposed control system is addressed in the following theorem.

Theorem 2 Consider the dynamic system (1) and the observer (5). Let the perturbation estimation error $\Delta \tilde{\mathbf{p}}_{2}(t, \mathbf{x}, \mathbf{u})$ be defined as (27). Suppose that

1)

$\left\|\Delta \tilde{\mathbf{p}}_{2}(t, \mathbf{x}, \mathbf{u})\right\| \leq c_{2}$

in accordance with (28), where $c_{2}$ is an unknown positive constant;

2) the initial condition $\mathbf{e}(0)$ satisfies the following inequality

$\left\|\mathbf{B}^{g} \mathbf{e}(0)\right\| \leq \frac{k_{b}}{\|\mathbf{B}\|}$, 
and the initial condition $\hat{\mathbf{z}}(0)$ fulfills the following inequality

$\left\|\mathbf{e}_{\hat{z}}(0)\right\|=\left\|\hat{\mathbf{z}}(0)-\mathbf{W}^{g} \mathbf{x}_{d}(0)\right\|<\frac{k_{b}}{\|\mathbf{W}\|}$.

If the controller (19) with adaptive laws (23) and (24) are employed, then

(a) the states $\mathbf{z}$ and $\mathbf{x}$ are all bounded; both $\sigma$ and $\mathbf{e}_{\hat{z}}$ will reach zero within a finite time. Moreover, not only $\|\mathbf{e}(t)\|<$ $2 k_{b}+\|\mathbf{W} \tilde{\mathbf{z}}\|$ for all time, but also $\mathbf{e}(t)$ will approach zero in a finite time;

(b) $\eta_{2}$ and adaptive gain $\hat{c}_{2}$ are all bounded for all time;

(c) the nominal signal $\sigma_{(\text {nom })}$ and the perturbation estimation signal $\mathbf{p}_{\text {est }}(t)$ are all bounded for all time.

Proof: Substituting (20) into (18) yields

$\dot{\sigma}(t)=-\varepsilon \sigma(t)+\mathbf{B}^{g} \Phi_{2} \mathbf{z}(t)+\mathbf{B}^{g} \Delta \mathbf{p}+\mathbf{u}_{s}-\eta_{2}-\mathbf{p}_{\text {est }}$.

Define a Barrier Lyapunov function candidate as

$$
\begin{aligned}
V= & \frac{k_{b}^{2}}{\|\mathbf{W}\|^{2} \pi} \tan \theta_{1}\left(\mathbf{e}_{\hat{z}}\right)+\frac{2 k_{b}}{\|\mathbf{B}\| \pi} \tan \theta_{2}(\sigma)+\frac{1}{2} \tilde{c}_{2}^{2}(t) \\
& +\left\|\eta_{2}(t)\right\|,
\end{aligned}
$$

where $\tilde{c}_{2}(t) \triangleq \hat{c}_{2}(t)-c_{2}$ is adaptive error of unknown positive constant $c_{2}$, and $\eta_{2}(t)$ is a state variable defined in (24). All the possible cases that may occur when computing the time derivative of $V$ are analyzed as follows.

Case 1: $\mathbf{e}_{\hat{z}} \neq \mathbf{0}, \sigma \neq \mathbf{0}$, and $\eta_{2} \neq \mathbf{0}$

By using (27), one can compute the time derivative of (31) along the trajectory of (17) and (30) as

$$
\begin{aligned}
\dot{V} \leq & \left\|\mathbf{e}_{\hat{z}}\right\|\left\|\mathbf{W}^{g} \Phi_{1}\right\|\|\mathbf{s}(t)\| \sec ^{2} \theta_{1}+\left\|\mathbf{e}_{\hat{z}}\right\|\left\|\eta_{1}\right\| \sec ^{2} \theta_{1}-\tan \theta_{1} \\
& +\frac{\sigma^{T}(t)}{\|\sigma(t)\|}\left[-\varepsilon \sigma+\mathbf{u}_{s}\right] \sec ^{2} \theta_{2}+\left\|\Delta \tilde{\mathbf{p}}_{2}(t)\right\| \sec ^{2} \theta_{2} \\
& +\tilde{c}_{2}(t) \dot{\hat{c}}_{2}(t)+\frac{\eta_{2}^{T}(t)}{\left\|\eta_{2}(t)\right\|} \dot{\eta}_{2}
\end{aligned}
$$

By using (21) and (29), from (32) one is able to obtain

$$
\begin{aligned}
\dot{V} \leq & \left\|\mathbf{e}_{\hat{z}}\right\|\left\|\mathbf{W}^{g} \Phi_{1}\right\|\|\mathbf{s}(t)\| \sec ^{2} \theta_{1}+\left\|\mathbf{e}_{\hat{z}}\right\|\left\|\eta_{1}\right\| \sec ^{2} \theta_{1}-\tan \theta_{1} \\
& -\varepsilon\|\sigma\| \sec ^{2} \theta_{2}-\kappa-\tan \theta_{2}-\hat{c}_{2} \sec ^{2} \theta_{2}-\Psi_{2} \\
& +c_{2} \sec ^{2} \theta_{2}+\tilde{c}_{2}(t) \dot{\hat{c}}_{2}(t)+\frac{\eta_{2}^{T}(t)}{\left\|\eta_{2}(t)\right\|} \dot{\eta}_{2} \\
= & -\varepsilon\|\sigma\| \sec ^{2} \theta_{2}-\kappa-\tilde{c}_{2}(t) \sec ^{2} \theta_{2}-\tan \theta_{1}-\tan \theta_{2} \\
& +\tilde{c}_{2}(t) \dot{\hat{c}}_{2}(t)+\frac{\eta_{2}^{T}(t)}{\left\|\eta_{2}(t)\right\|} \dot{\eta}_{2} .
\end{aligned}
$$

Substituting (23) and (24) into (33) further simplifies (33) as

$$
\begin{aligned}
\dot{V} & \leq-\varepsilon\|\sigma\| \sec ^{2} \theta_{2}(\sigma)-\tan \theta_{1}\left(\mathbf{e}_{\bar{z}}\right)-\tan \theta_{2}(\sigma)-\gamma \\
& <-\gamma<0 .
\end{aligned}
$$

According to (34), it can be seen that $V$ is bounded and its magnitude decreases. It also implies that $\sigma, \mathbf{e}_{\hat{z}}, \tilde{c}_{2}$, and $\eta_{2}$ are all bounded in this case.
Case 2: $\mathbf{e}_{\hat{z}} \neq \mathbf{0}, \sigma=\mathbf{0}$, and $\eta_{2} \neq \mathbf{0}$

In this case $V=k_{b}^{2} \tan \theta_{1}\left(\mathbf{e}_{\hat{z}}\right) /\left(\|\mathbf{W}\|^{2} \pi\right)+\left\|\eta_{2}(t)\right\|+\tilde{c}_{2}^{2}$ $/ 2$. By using (17) and (24), one computes the time derivative of $V$ as

$$
\begin{aligned}
\dot{V} \leq & \left\|\mathbf{e}_{\hat{z}}\right\|\left\|\mathbf{W}^{g} \Phi_{1}\right\|\|\mathbf{s}(t)\| \sec ^{2} \theta_{1}+\left\|\mathbf{e}_{\hat{z}}\right\|\left\|\eta_{1}\right\| \sec ^{2} \theta_{1}-\tan \theta_{1} \\
& \left.-\gamma-\Psi_{2}, \mathbf{s}\right) \\
= & -\tan \theta_{1}-\gamma \leq-\gamma<0 .
\end{aligned}
$$

Hence $V$ is bounded and the magnitude of $V$ will decrease, which also indicates that the magnitude of $\mathbf{e}_{\hat{z}}, \eta_{2}$ and $\tilde{c}_{2}$ are bounded in this case.

Case 3: $\mathbf{e}_{\hat{z}}=\mathbf{0}, \sigma \neq \mathbf{0}$, and $\eta_{2} \neq \mathbf{0}$

In this case $V=2 k_{b} \tan \theta_{2}(\sigma) /(\|\mathbf{B}\| \pi)+\tilde{c}_{2}^{2} / 2+\left\|\eta_{2}\right\|$. By using (27) and (29) one is able to compute the time derivative of $V$ along the trajectory of (30) as

$$
\begin{aligned}
\dot{V}= & \frac{\sigma^{T}(t)}{\|\sigma(t)\|}\left[-\varepsilon \sigma+\mathbf{u}_{s}+\Delta \tilde{\mathbf{p}}_{2}\right] \sec ^{2} \theta_{2}+\tilde{c}_{2}(t) \dot{\tilde{c}}_{2}(t) \\
& +\frac{\eta_{2}^{T}(t)}{\left\|\eta_{2}(t)\right\|} \dot{\eta}_{2} \\
\leq & \frac{\sigma^{T}(t)}{\|\sigma(t)\|}\left[-\varepsilon \sigma+\mathbf{u}_{s}\right] \sec ^{2} \theta_{2}+c_{2} \sec ^{2} \theta_{2}+\tilde{c}_{2}(t) \dot{\hat{c}}_{2}(t) \\
& +\frac{\eta_{2}^{T}(t)}{\left\|\eta(t)_{2}\right\|} \dot{\eta}_{2} .
\end{aligned}
$$

Substituting (21), (23) and (24) into (36) yields

$$
\begin{aligned}
\dot{V} & \leq-\varepsilon\|\sigma\| \sec ^{2} \theta_{2}(\sigma)-\kappa-\tan \theta_{2}(\sigma)-\gamma-2 \Psi_{2}\left(\mathbf{e}_{\hat{z}}, \mathbf{s}\right) \\
& \leq-\tan \theta_{2}(\sigma)-\gamma \leq-\gamma<0 .
\end{aligned}
$$

Equation (37) also implies that $V$ is bounded and will continuously decrease. Therefore, $\sigma, \tilde{c}_{2}$ and $\eta_{2}$ are all bounded in this case.

Case 4: $\mathbf{e}_{\hat{z}} \neq \mathbf{0}, \sigma \neq \mathbf{0}, \eta_{2}=\mathbf{0}$

In this case $V=k_{b}^{2} \tan \theta_{1}\left(\mathbf{e}_{\hat{z}}\right) /\left(\|\mathbf{W}\|^{2} \pi\right)+2 k_{b} \tan \theta_{2}(\sigma) /$ $(\|\mathbf{B}\| \pi)+\tilde{c}_{2}^{2}(t) / 2$. By using (23), from (33) one can obtain

$$
\begin{aligned}
\dot{V} & \leq-\varepsilon\|\sigma\| \sec ^{2} \theta_{2}(\sigma)-\kappa-\tan \theta_{1}\left(\mathbf{e}_{\hat{z}}\right)-\tan \theta_{2}(\sigma) \\
& \leq-\kappa-\tan \theta_{1}\left(\mathbf{e}_{\hat{z}}\right)-\tan \theta_{2}(\sigma) \leq-\kappa<0 .
\end{aligned}
$$

Therefore, $V$ is bounded and its magnitude decreases, which means that the magnitude of $\mathbf{e}_{\hat{z}}, \sigma$ and $\tilde{c}_{2}$ are bounded in this case.

Case 5: $\mathbf{e}_{\hat{z}}=\mathbf{0}, \sigma \neq \mathbf{0}$, and $\eta_{2}=\mathbf{0}$

In this case $V=2 k_{b} \tan \theta_{2}(\sigma) /(\|\mathbf{B}\| \pi)+\tilde{c}_{2}^{2}(t) / 2$. By using equations (30), (27) and (29), one computes the time derivative of $V$ as

$$
\dot{V} \leq \frac{\sigma^{T}(t)}{\|\sigma(t)\|}\left[-\varepsilon \sigma+\mathbf{u}_{s}\right] \sec ^{2} \theta_{2}+c_{2} \sec ^{2} \theta_{2}+\tilde{c}_{2}(t) \dot{\hat{c}}_{2}(t) \text {. }
$$

Substituting (21) and (23) into (39) yields

$\dot{V} \leq-\tan \theta_{2}-\kappa \leq-\kappa<0$. 
Equation (40) clearly indicates that $V$ is bounded, and the value of $V$ will decrease. Hence $\sigma$ and adaptive error $\tilde{c}_{2}(\mathrm{t})$ are all bounded in this case.

Case 6: $\mathbf{e}_{\hat{z}} \neq \mathbf{0}, \sigma=\mathbf{0}, \eta_{2}=\mathbf{0}$

In this case $V=k_{b}^{2} \tan \theta_{1}\left(\mathbf{e}_{\hat{z}}\right) /\left(\|\mathbf{W}\|^{2} \pi\right)+\tilde{c}_{2}^{2}(t) / 2$. By using (23), one computes the time derivative of $V$ along the trajectory of (17) as

$\dot{V} \leq\left\|\mathbf{e}_{\hat{z}}\right\|\left\|\mathbf{W}^{g} \Phi_{1}\right\|\|\mathbf{s}\| \sec ^{2} \theta_{1}+\left\|\mathbf{e}_{\hat{z}}\right\|\left\|\eta_{1}\right\| \sec ^{2} \theta_{1}-\tan \theta_{1}$.

Equation (41) indicates that $\dot{V}$ may be greater or smaller than zero. However, from (24) it is seen that $\eta_{2}=\mathbf{0}$ and $\dot{\eta}_{2} \neq \mathbf{0}$, it reveals that the trajectory of $\eta_{2}$ will cross the surface $\eta_{2}=\mathbf{0}$ immediately (that is, $\eta_{2} \neq \mathbf{0}$ in the next time interval), and the status of system will switch to another case where $\eta_{2} \neq$ 0. Noted that the state $\mathbf{e}_{\hat{z}}$ is still bounded in this case due to the continuity of its trajectory in accordance with (17).

Case 7: $\mathbf{e}_{\bar{z}}=\mathbf{0}, \sigma=\mathbf{0}, \eta_{2} \neq \mathbf{0}$.

In this case the Lyapunov function becomes $V=\left\|\eta_{2}(t)\right\|$ $+\tilde{c}_{2}^{2}(t) / 2$. By using (23) and (24), one computes the time derivative of $V$ as

$\dot{V}=-\gamma-\Psi_{2}\left(\mathbf{e}_{\hat{z}}, \mathbf{s}\right) \leq-\gamma<0$.

According to (42), one can see that $V$ is a bounded function and the magnitude of $V$ will decrease. Hence one can conclude that $\eta_{2}$ and $\tilde{c}_{2}$ are bounded in this case.

Case 8: $\mathbf{e}_{\hat{z}}=\mathbf{0}, \sigma=\mathbf{0}, \eta_{2}=\mathbf{0}$.

In this case $V=\tilde{c}_{2}^{2} / 2$. Then $\dot{V}=0$ in accordance with (23). It indicates that the value of $V$ will stop decreasing in this case.

From the preceding analysis of cases $1-8$, it can be seen that

(a) $\dot{V}<0$ except in the cases 6 and 8 . Due to the quick switching ability of the proposed controller and the continuity of the functions in case 6 , the value of $V$ will still be bounded even if the occurrence of case 6 . The preceding analysis also reveals that the function $\mathbf{e}_{\hat{z}}$ and $\sigma$ will reach zero within a finite time. Hence the state variables $\mathbf{z}, \mathbf{s}$ and $\mathbf{x}$ are all bounded for all time in accordance with (6), (4) and (2).

On the other hand, from (22) and (7) it is seen that if the state variable $\sigma$ approaches the boundary $k_{b} /\|\mathbf{B}\|$ or the state variable $\mathbf{e}_{\hat{\mathbf{z}}}$ approaches the boundary $k_{b} /\|\mathbf{W}\|$, then both $\theta_{1}$ and $\theta_{2}$ approach $\pi / 2$, it also implies that $V \rightarrow \infty$, which will not happen in accordance with the previous analysis. Hence if $\sigma(0)$ and $\mathbf{e}_{\hat{z}}(0)$ fulfill $\|\sigma(0)\|<k_{b} /\|\mathbf{B}\|$ and $\left\|\mathbf{e}_{\hat{z}}(0)\right\|<$ $k_{b} /\|\mathbf{W}\|$ respectively, then $\left\|\mathbf{B}^{g} \mathbf{e}(t)\right\|<k_{b} /\|\mathbf{B}\|,\left\|\mathbf{e}_{\hat{z}}(t)\right\|<$ $k_{b} /\|\mathbf{W}\|$ for all time, and the Lyapunov function $V$ will not approach infinity. By using the fact that $\mathbf{z}-\mathbf{W}^{g} \mathbf{x}_{d}=\tilde{\mathbf{z}}+\mathbf{e}_{\hat{z}}$, from (2) one can obtain

$\mathbf{M}^{-1} \mathbf{e}(t)=\left[\begin{array}{c}\tilde{\mathbf{z}}+\mathbf{e}_{\hat{z}}(t) \\ \sigma(t)\end{array}\right]$ in accordance with (4). Equation (43) also implies that $\mathbf{e}(t)$ $=\mathbf{W}\left(\mathbf{e}_{\hat{z}}+\tilde{\mathbf{z}}\right)+\mathbf{B} \sigma$, which further implies $\|\mathbf{e}(t)\| \leq\|\mathbf{W}\|\left\|\mathbf{e}_{\hat{\mathbf{z}}}\right\|$ $+\|\mathbf{W} \tilde{\mathbf{z}}\|+\|\mathbf{B}\|\|\sigma\|<2 k_{b}+\|\mathbf{W} \tilde{\mathbf{z}}\|, \forall t$. Since $\tilde{\mathbf{z}}, \mathbf{e}_{\hat{\mathbf{z}}}$ and $\sigma$ will tend to zero in a finite time, $\mathbf{e}(t)$ will also reach zero within a finite time.

(b) The adaptive gain $\hat{c}_{2}$ is monotonically increasing and bounded above in accordance with (23) and analysis in part (a). Therefore, there exits a finite constant $c_{2 \infty}$ such that $\lim _{t \rightarrow \infty} \hat{c}_{2}=c_{2 \infty}$ [37]. Since $\eta_{2}^{T} \dot{\eta}_{2} \leq 0$ in (24), the signal $\eta_{2}$ is bounded.

(c) According to the previous analysis, one can see that the states $\mathbf{z}, \mathbf{s}$ and $\mathbf{x}$ are all bounded. From (30) and (25) one can obtain

$\dot{\sigma}-\dot{\sigma}_{(\text {пот })}=-\varepsilon\left[\sigma-\sigma_{(\text {пот })}\right]+\mathbf{B}^{g} \Phi_{2} \mathbf{z}+\mathbf{B}^{g} \Delta \mathbf{p}-\eta_{2}(t)$.

It is seen that the term $\mathbf{B}^{g} \Phi_{2} \mathbf{z}(t)+\mathbf{B}^{g} \Delta \mathbf{p}-\eta_{2}$ in (44) is also bounded. Hence according to (44), one can see that $\sigma-\sigma_{\text {nom }}$ is bounded since $\varepsilon>0$. Therefore, $\sigma_{\text {nom }}$ must be bounded, and from (26) the perturbation estimation signal $\mathbf{p}_{\text {est }}$ must be bounded due to $\hat{\mathbf{p}}(t)$ is bounded.

\section{The Effects of Designed Parameters on System's Performance}

It is very important to know the impact of each designed parameter on system's performance, this is analyzed as follows.

(a) $\rho_{1}$ : According to (16), one is able to see that choosing larger value of $\rho_{1}$ will cause $V_{1}$ decreasing rapidly. It also implies that the estimation error $\tilde{\mathbf{z}}$ will approach zero more faster.

(b) $\varepsilon$ : If larger value of $\varepsilon$ is chosen, the convergent rate of $\sigma_{\text {nom }}$ and $\sigma$ will become faster in accordance with (25) and (34) respectively, but it may increase the control input energy in accordance with (20).

(c) $\kappa$ : From (38), one can see that larger value of $\kappa$ may drive the trajectory of $\sigma$ to zero more faster. However, it will also increase the magnitude and the chattering phenomenon of control input $\mathbf{u}$ in accordance with (21).

(d) $\gamma$ : According to (34), (35), (37) and (42), choosing larger value of $\gamma$ will make the values of $V, \sigma, \mathbf{e}_{\hat{z}}$, and $\eta_{2}$ decreasing rapidly, but it may result in larger control effort in accordance with (24).

(e) $\varphi_{1}$ and $\varphi_{2}$ : The purpose of designing $\varphi_{i}, i=1,2$ in (8) and (24) is to force the trajectories of $\eta_{i}, i=1,2$ to leave the surface $\eta_{i}=\mathbf{0}$. Thus, only a $\varphi_{i}$ with small entries is enough to achieve this goal. 


\section{Numerical Example}

Consider a perturbed nonlinear system with dynamic equation given by (1), where the known vector and matrices $\mathbf{f}(\mathbf{x})$, $\mathbf{B}, \mathbf{C}$, are

$\mathbf{f}(\mathbf{x})=\left[\begin{array}{c}x_{3} x_{2} \\ x_{4} \cos \left(x_{3}\right) \\ x_{3} \sin \left(x_{2}\right) \\ x_{1}^{2} x_{3}\end{array}\right], \mathbf{B}=\left[\begin{array}{ll}0 & 0 \\ 1 & 0 \\ 0 & 1 \\ 0 & 0\end{array}\right], \quad \mathbf{C}=\left[\begin{array}{llll}0 & 1 & 1 & 0 \\ 1 & 0 & 1 & 0 \\ 1 & 1 & 0 & 0\end{array}\right]$,

and $\mathbf{x} \triangleq\left[x_{1}, x_{2}, x_{3}, x_{4}\right]^{T}$. The vector $\mathbf{u} \triangleq\left[u_{1}, u_{2}\right]^{T}$ represents the control input. For demonstrating the robustness and effectiveness of the proposed control scheme by using computer simulation, we assume that the unknown perturbation $\Delta \mathbf{p}(t, \mathbf{x}, \mathbf{u})$ is

$\Delta \mathbf{p}=\left[\begin{array}{c}-2 x_{1}^{2}+x_{2} \sin (2 t) \\ x_{3} x_{1} \sin (t) \\ x_{2} \sin \left(x_{3}\right) \\ x_{4}^{2} \cos (2 t)\end{array}\right]+\left[\begin{array}{cc}x_{1} \sin (t) & \sin \left(x_{3} x_{1} t\right) \\ x_{3} x_{1} & x_{3} \sin \left(x_{2}\right) \\ 0 & x_{1} x_{2} \\ x_{4} \sin (2 t) & x_{1} x_{3}\end{array}\right] \mathbf{u}$.

According to Section 3, we design the matrix $\mathbf{W}$ and $\mathbf{F}$ respectively as

$\mathbf{W}=\left[\begin{array}{llll}1 & 0 & 0 & 0 \\ 0 & 0 & 0 & 1\end{array}\right]^{T}, \quad \mathbf{F}=\left[\begin{array}{ccc}1 / 2 & -1 / 2 & 1 / 2 \\ 1 / 2 & 1 / 2 & -1 / 2\end{array}\right]$.

The functions $\Phi_{1}(\mathbf{s})$ and $\Phi_{2}(\mathbf{s}, \mathbf{z})$ are chonsen as

$\Phi_{1}(\mathbf{s})=\left[\begin{array}{cc}s_{2} & 0 \\ 0 & 0 \\ 0 & \sin \left(s_{1}\right) \\ 0 & 0\end{array}\right], \Phi_{2}(\mathbf{s}, \mathbf{z})=\left[\begin{array}{cc}0 & 0 \\ 0 & \cos \left(s_{2}\right) \\ 0 & 0 \\ z_{1} s_{2} & 0\end{array}\right]$,

where $s_{1}=x_{2}, s_{2}=x_{3}$ in accordance with (2), which are measurable, whereas the state variables $x_{1}=z_{1}$ and $x_{4}=z_{2}$ are unmeasurable in this example. The tracking error vector $\mathbf{e}(t)$ is required to fulfill $\|\mathbf{e}(t)\|<4+\|\mathbf{W} \tilde{\mathbf{z}}\|$ for all time, which implies $k_{b}=2$. The tracking signal is assumed to be $\mathbf{x}_{d}=[\cos (t), \sin (t), \cos (2 t), 0]^{T}$, and the nonlinear positive functions $\beta(\hat{\mathbf{x}})$ and $\zeta(\mathbf{u})$ in Theorem 1 are given by

$\beta(\hat{\mathbf{x}})=2\|\hat{\mathbf{x}}\|, \quad \zeta(\mathbf{u})=\|\mathbf{u}\|$.

The adaptive sliding mode tracking controller as well as partial state observer are designed in accordance with (19) and (5) respectively. The designed parameters are chosen to be $\left(\rho_{1}, \varphi_{1}, \varphi_{2}, h_{1}, \varepsilon, \kappa, \gamma\right)=\left(1,[2,2]^{T},[2,2]^{T}, 0.2,4,2,1\right)$ respectively. Fig. 1 to Fig. 7 show the results of computer simulation with step time $0.1 \mathrm{msec}$, the unknown initial condition is assumed to be $\mathbf{x}(0)=[0.1,-0.2,0.5,-0.3]^{T}$. Fig. 1 indicates that the trajectory of estimation error $\tilde{\mathbf{z}}$ approaches zero in $5.8 \mathrm{sec}$. All the tracking errors, as shown in Fig. 2, converge to zero before $6 \mathrm{sec}$. The trajectory of $\|\mathbf{e}(t)\|$ is depicted in Fig. 3, it converges to zero before $6 \mathrm{sec}$., and satisfies the required constraint $\|\mathbf{e}(t)\|<4+\|\mathbf{W} \tilde{\mathbf{z}}\|$ for all time. The trajectories of adaptive gains $\hat{c}_{0}, \hat{c}_{1}$ and $\hat{c}_{2}$ are illustrated in Fig. 4, they are all bounded and reach a finite limit respectively. The state variables $\eta_{1}$ and $\eta_{2}$, as illustrated from Fig. 5 to Fig. 6, are all bounded. Noted that if $\eta_{i j}=0$, then $\dot{\eta}_{i j} \neq 0$ in the first three seconds, $i, j=1,2$ for avoiding the occurrence of case 6 . The control input $\mathbf{u}$, as displayed in Fig. 7, is bounded, but it reveals the chattering phenomenon. Noted that the perturbation estimation signal $\mathbf{p}_{\text {est }}(t)$ and the nominal signal $\sigma_{\text {nom }}(t)$ are all bounded for all time.

If the requirement of $\|\mathbf{e}\|$ changes to $\|\mathbf{e}(t)\|<2+\|\mathbf{W} \tilde{\mathbf{z}}\|$, then $k_{b}=1$; which will affect the control input in accordance with (20). The design parameters $\left(\rho_{1}, \varphi_{1}, \varphi_{2}, h_{1}, \varepsilon, \kappa, \gamma\right)$ are not affected by changing the value of $k_{b}$. The computer simulations with $k_{b}=1$ are illustrated from Fig. 8 to Fig. 9. Fig. 8 confirms that the requirement $\|\mathbf{e}(t)\|<2+\|\mathbf{W} \tilde{\mathbf{z}}\|$ is not violated for all time, and $\|\mathbf{e}(t)\|$ reaches zero in $6.3 \mathrm{sec}$. Fig. 9 reveals that the control energy increases when smaller $k_{b}$ is given.

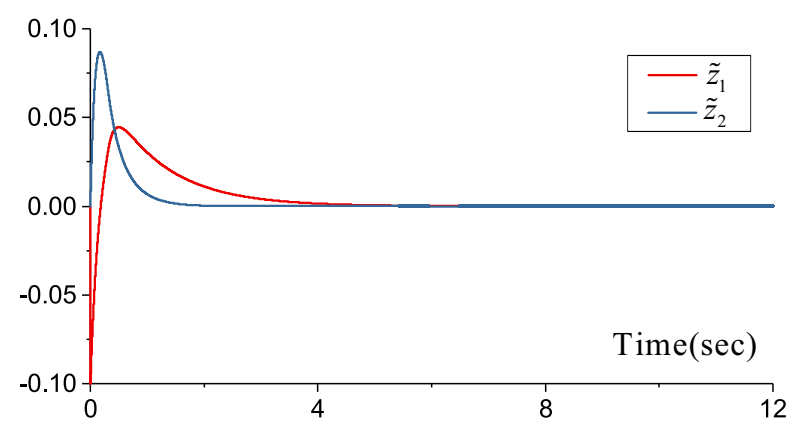

Fig. 1 The trajectory of estimation error $\tilde{\mathbf{z}}$ with $k_{b}=2, \kappa=2$.

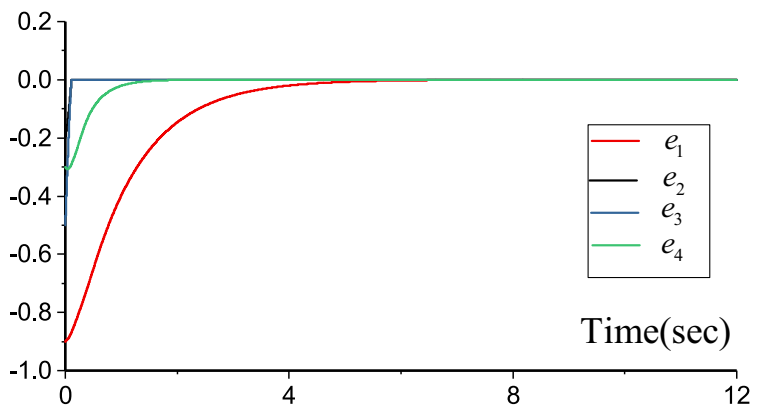

Fig. 2 The trajectory of tracking error $\mathbf{e}(t)$.

\section{Conclusions}

An adaptive sliding mode tracking controller and a partial states observer are successfully proposed in this paper for a class of perturbed nonlinear systems with state constraints. This research results clearly shows that the proposed control 


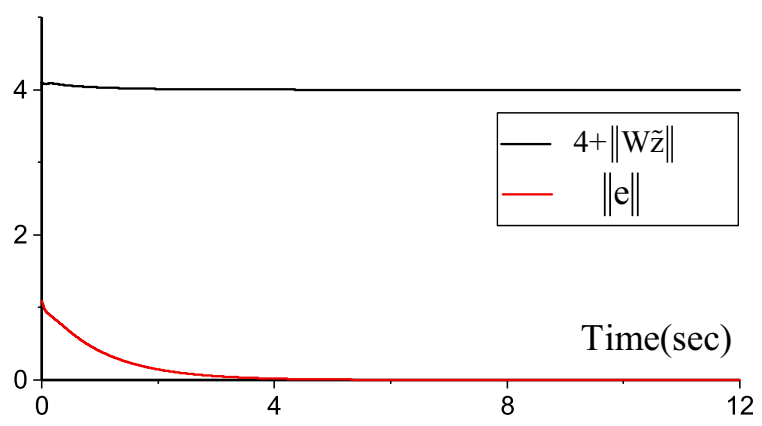

Fig. 3 The trajectory of $\|\mathbf{e}\|, k_{b}=2$.

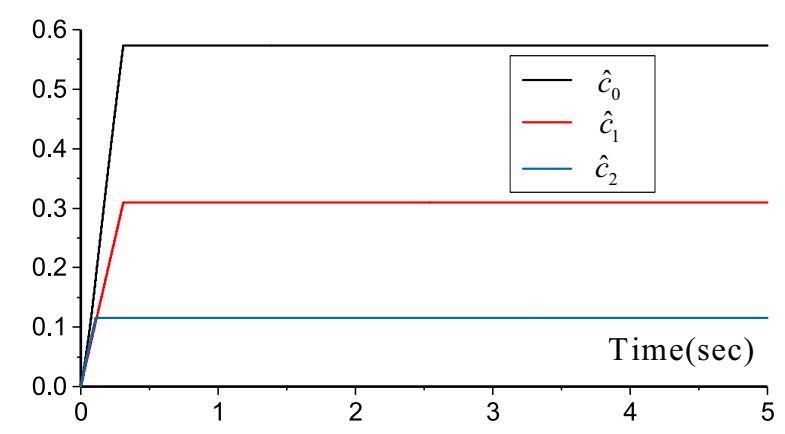

Fig. 4 The trajectories of adaptive gains.

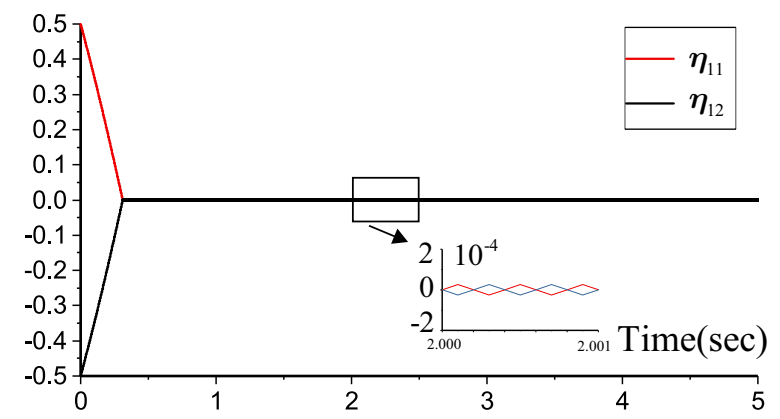

Fig. 5 The trajectory of $\eta_{1}$.

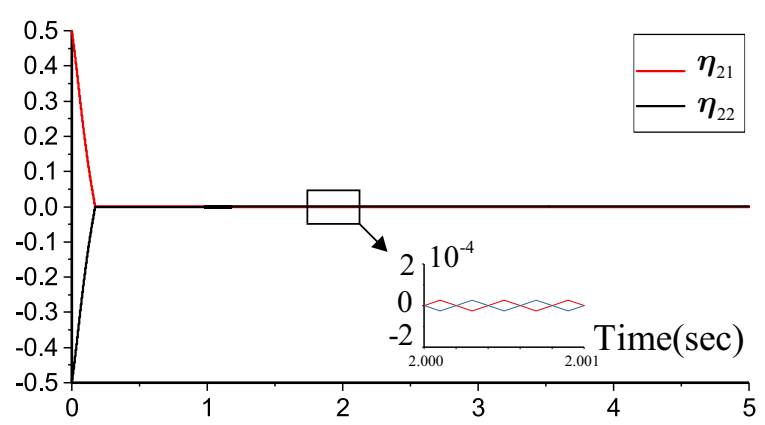

Fig. 6 The trajectory of $\eta_{2}$.

strategy has the following advantages: (1) Some state variables do not need to be measurable, and all the state tracking errors can converge to zero within a finite time. (2) It has the ability to constrain the magnitude of tracking error. (3) The dynamic equations of the plant do not need to be in strict feedback form. (4) There is no need to know the upper bounds of both match and unmatched perturbations in

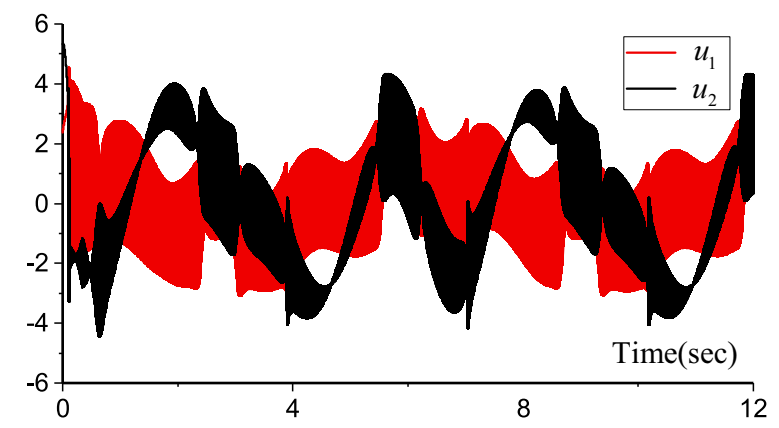

Fig. 7 The trajectory of control input $\mathbf{u}$ with $k_{b}=2, \kappa=2$.

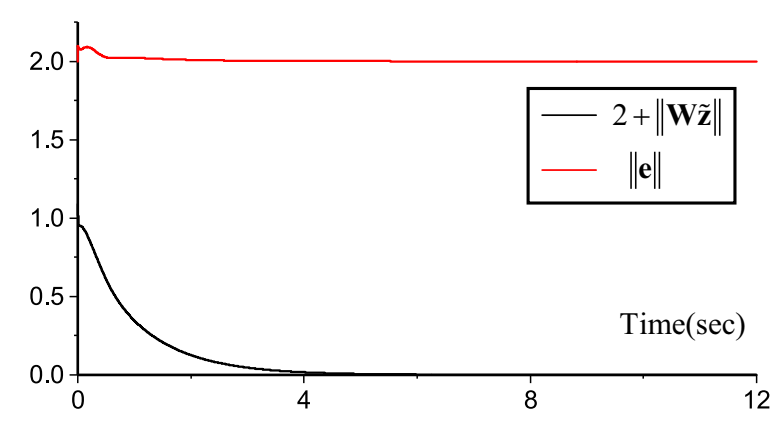

Fig. 8 The trajectory of $\|\mathbf{e}\|, k_{b}=1$.

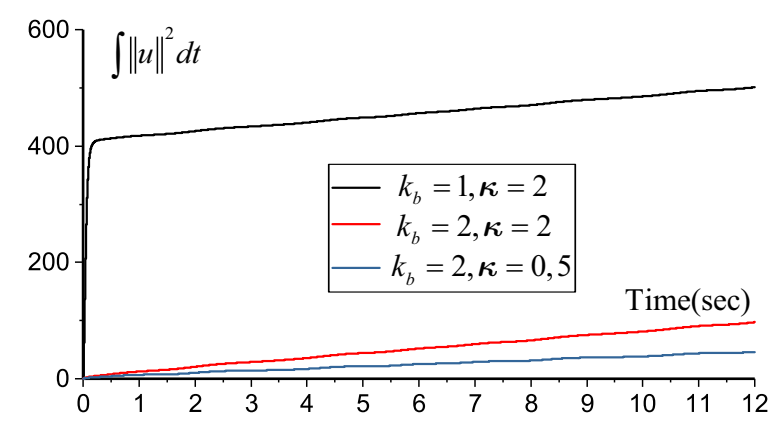

Fig. 9 Control energy with different $k_{b}$ and $\kappa$.

advance. (5) It can be applied to MIMO systems. For future study, reducing chattering phenomenon of the control input is worth considering.

\section{Conflict of Interest}

The authors declare that they have no conflict of interest.

\section{Data Availability Statement}

Data sharing is not applicable to this article as no datasets were generated or analyzed during this study.

\section{References}

1. Shen, J., Lam, J.: Static output-feedback stabilization with optimal L1-gain for positive linear systems. Automatica. 63, 248-253 (2016) 
2. Liu, X., Sun, X., Liu, S., Xu S., Cai, M.: Design of robust slidingmode output-feedback control with suboptimal guaranteed cost. IET Control Theory \& Applic. 9(2), 232-239 (2015)

3. Yan, X.-G., Spurgeon, S.K., Edwards, C.: Memoryless static output feedback sliding mode control for nonlinear systems with delayed disturbances. IEEE Trans. Autom. Control. 59(7), 1906-1912(2014)

4. Chang J.-L., Wu, T.-C.: Output feedback variable structure control design for uncertain nonlinear Lipschitz systems. J. Control Science and Engineering. 2015(51), (2015)

5. Fei, J., Chu, Y.: Double hidden layer output feedback neural adaptive global sliding mode control of active power filter. IEEE Trans. Power Electron. 35(3), 3069-3084 (2020)

6. Li, Y., Hua, C., Guan, X.: Distributed output feedback leaderfollowing control for high-order nonlinear multiagent system using dynamic gain method. IEEE Trans. Cybern. 50(2), 640-649 (2020)

7. Li, Y.-X., Yang, G.-H.: Fuzzy adaptive output feedback faulttolerant tracking control of a class of uncertain nonlinear systems with nonaffine nonlinear faults. IEEE Trans. Fuzzy Syst. 24(1), 223234 (2016)

8. Park, J.-H., Kim, S.-H., Park, T.-S.: Output-feedback adaptive neural controller for uncertain pure-feedback nonlinear systems using a high-order sliding mode observer. IEEE Trans. Neural Netw. Learn. Syst. 30(5), 1596-1601 (2019).

9. Wang, M., Wang, Z., Chen, Y., Sheng, W.: Observer-based fuzzy output-feedback control for discrete-time strict-feedback nonlinear systems with stochastic noises. IEEE Trans. Cybern. 50(8), 37663777 (2020)

10. Choi, Y.H., Yoo, S.J.: Filter-driven-approximation-based control for a class of pure-feedback systems with unknown nonlinearities by state and output feedback. IEEE Trans. Syst., Man, Cybern., Syst. 48(2), 161-176 (2018)

11. Huang, J.-T.: Adaptive fuzzy state/output feedback control of nonstrict-feedback systems: A direct compensation approach. IEEE Trans. Cybern. 49(6), 2046-2059 (2019)

12. Lei, J., Khalil, H. K.: High-gain-predictor-based output feedback control for time-delay nonlinear systems. Automatica. 71, 324-333 (2016)

13. Laila, D. S., Gruenbacher, E.: Nonlinear output feedback and periodic disturbance attenuation for setpoint tracking of a combustion engine test bench. Automatica. 64, 29-36 (2016)

14. Danielson, C., Berntorp, K., Weiss, A., Cairano, S.D.: Robust motion planning for uncertain systems with disturbances using the invariant-set motion planner. IEEE Trans. Autom. Control. 65(10), 4456-4463 (2020)

15. Preindl, M.: Robust control invariant sets and Lyapunov-based MPC for IPM Synchronous motor drives. IEEE Trans. Ind. Electron. 63(6), 3925-3933 (2016)

16. Weiss, A., Baldwin, M., Erwin, R.S., Kolmanovsky, I.: Model predictive control for spacecraft rendezvous and docking: Strategies for handling constraints and case studies. IEEE Trans. Control Syst. Technol. 23(4), 1638-1647 (2015)

17. Tarczewski, T., Grzesiak, L.M.: Constrained state feedback speed control of PMSM based on model predictive approach. IEEE Trans. Ind. Electron. 63(6), 3867-3875 (2016)

18. Tang, X., Deng, L., Liu, N., Yang, S., Yu, J.: Observer-based output feedback MPC for TVS fuzzy system with data loss and bounded disturbance. IEEE Trans. Cybern. 49(6), 2119-2132 (2019)

19. Hosseinzadeh, M., Garone, E.: An explicit reference governor for the intersection of concave constraints," IEEE Trans. Autom. Control. 65(1), 1-11 (2020)

20. Xu, Z., Li, L., Yao, J., Hu, X., Liu, Q., Xie, N.: State constraint control for uncertain nonlinear systems with disturbance compensation. IEEE Access. 7, 155251-155261 (2019).

21. Jing, Y.-H., Yang, G.-H.: Adaptive fuzzy output feedback faulttolerant compensation for uncertain nonlinear systems with infinite number of time-varying actuator failures and full-state constraints. IEEE Trans. Cybern. 51(2), 568-578 (2021).
22. Wang, W., Tong, S.: Adaptive fuzzy containment control of nonlinear strict-feedback systems with full state constraints. IEEE Trans. Fuzzy Syst. 27(10), 2024-2038 (2019)

23. Tran, D.T., JIN, M., Ahn, K.K.: Nonlinear extended state observer based on output feedback control for a manipulator with timevarying output constraints and external disturbance. IEEE Access. 7, 156860-156870 (2019)

24. Liu, N., Shao, X., Li, J., Zhang, W.: Attitude restricted backstepping anti-disturbance control for vision based quadrotors with visibility constraint. ISA Trans. 100, 109-125 (2020)

25. Yu, J., Zhao, L., Yu, H., Lin, C.: Barrier Lyapunov functions-based command filtered output feedback control for full-state constrained nonlinear systems. Automatica. 105, 71-79 (2019)

26. Zhang, Z., Wu, Y.: Adaptive fuzzy tracking control of autonomous underwater vehicles with output constraints. IEEE Trans. Fuzzy Syst. 29(5), 1311-1319(2021)

27. Liu, Y.-J., Gong, M., Tong, S., Chen, C.L.P., Li, D.-J.: Adaptive fuzzy output feedback control for a class of nonlinear systems with full state constraints. IEEE Trans. Fuzzy Syst. 26(5), 2607-2617 (2018)

28. Wan, M., Yin, Y.: Adaptive dynamic surface control based on observer for switched non-strict feedback systems with full state constraints. IEEE Access. 8, 71008-71020 (2020)

29. Su, Q., Wan, M.: Adaptive neural dynamic surface output feedback control for nonlinear full states constrained systems. IEEE Access. 8, 131590-131600 (2020)

30. Zhang, J., Li, K., Li, Y.: Output-feedback based simplified optimized backstepping control for strict-feedback systems with input and state constraints. IEEE/CAA J. Automatica Sinica. 8(6), 1119$1132(2021)$

31. Zhang, S., Dong, Y., Ouyang, Y., Yin, Z., Peng, K.: Adaptive neural control for robotic manipulators with output constraints and uncertainties. IEEE Trans. Neural Netw. Learn. Syst. 2911, 55545564 (2018)

32. He, W., Kong, L., Dong, Y., Yu, Y., Yang, C., Sun, C.: Fuzzy tracking control for a class of uncertain MIMO nonlinear systems with state constraints. IEEE Trans. Syst., Man, Cybern., Syst.. 49(3), 543-554 (2019).

33. He, W., Yin, Z., Sun, C.: Adaptive neural network control of a marine vessel with constraints using the asymmetric barrier Lyapunov function. IEEE Trans. Cybern. 47(7), 1641-1651 (2017)

34. Xia, G., Sun, C., Zhao, B., Xia, X., Sun, X.: Neuroadaptive distributed output feedback tracking control for multiple marine surface vessels with input and output constraints. IEEE Acess. 7, 123076123085 (2019).

35. Chiang, Y.-C., Cheng, C.-C.: Terminal adaptive output feedback variablestructure control. IET Control Theory \& Applic. 12(10), 1376-1383 (2018)

36. Sohrab, H.H.: Basic Real Analysis (2nd ed.), Birkhäuser, Springer, New York (2003)

37. Tao, G.: Adaptive control design and analysis. John Wiley \& Sons, New Jersey (2003)

38. Cheng, C.-C., Chang, M.-W.: Design of derivative estimator using adaptive sliding mode technique. In: American Control Conference, Minneapolis, pp. 2611-2615 (2006) 